\title{
The association between aspirin resistance and extent and severity of coronary atherosclerosis
}

\author{
Serkan Kahraman, ${ }^{1}$ (D) Ali Dogan, ${ }^{2}$ (D) Murat Ziyrek, ${ }^{1}$ (D) Emrah Usta, ${ }^{3}$ (D) Onder Demiroz, ${ }^{4}$ (D) Cavlan Ciftci ${ }^{4}$ \\ ${ }^{1}$ Department of Cardiology, Istanbul Silivri State Hospital, Istanbul, Turkey \\ 2Department of Cardiology, Istanbul Yeniyuzyil University Faculty of Medicine, Gaziosmanpasa Hospital, Istanbul, Turkey \\ ${ }^{3}$ Department of Cardiology, Baltalimani Training and Research Hospital, Istanbul, Turkey \\ ${ }^{4}$ Department of Cardiology, Istanbul Bilim University Faculty of Medicine, Istanbul, Turkey
}

\begin{abstract}
OBJECTIVE: Uncontrolled inflammatory responses could contribute to the pathogenesis of many leading causes of human morbidity and mortality. Aspirin is an anti-inflammatory and antithrombotic drug that is used in the primary and secondary protection in atherothrombotic diseases and complications. The aim of the present study was to analyze the effect of aspirin resistance on the extent and severity of atherosclerosis.
\end{abstract}

METHODS: One hundred patients who underwent coronary angiography with suspected or known coronary artery disease and were using aspirin were enrolled in the study.

RESULTS: Of these 100 patients, 30 ( 8 female and 22 male) formed the aspirin-resistant group (ARG), and 70 (22 female and 48 male) formed the control group. Gensini scoring system (GSS) was significantly higher in the ARG than in the control group (80.5 (36-166) vs. $45(2-209) ; \mathrm{p}<0.001)$. The number of percutaneous coronary intervention (PCI) patients was significantly higher in the ARG (13 of $30(43.3 \%)$ ARG vs. 13 of $70(18.6 \%)$ control group; $p=0.01)$. Furthermore, when we evaluate the 16 reintervention patients, stent restenosis was significantly higher in the ARG (11 of 16 (68.75\%) ARG vs. 5 of $16(31.25 \%)$ control group; $p=0.016)$. Multivariate logistic regression analysis revealed that GSS ( $p=0.038 ; 95 \%$ CI: $1.001-1.026$ ) and PCI history ( $p=0.017 ; 95 \%$ CI: $1.182-89.804$ ) were independent risk factors for aspirin resistance.

CONCLUSION: In conclusion, atherosclerotic burden as calculated by the GSS is significantly higher in aspirin-resistant patients. According to this result, we suggest that aspirin treatment can be prescribed in higher doses in aspirin resistance patients with coronary events. Furthermore, GSS and PCI history could be independent predictors of aspirin resistance.

Keywords: Aspirin resistance; atherosclerosis; coronary artery disease.

Cite this article as: Kahraman S, Dogan A, Ziyrek M, Usta E, Demiroz O, Ciftci C. The association between aspirin resistance and extent and severity of coronary atherosclerosis. North Clin Istanb 2018;5(4):323-328.

U ncontrolled inflammatory responses could contribute to the pathogenesis of many leading causes of human morbidity and mortality [1]. Atherosclerosis, a chronic low-grade inflammatory state, is one of the most common causes of death in developed countries and an example of uncontrolled inflammation [2]. The clinical importance of atherosclerosis attracts much attention to the inflammation cascade. Arachidonic acid is a polyunsaturated fatty acid that accounts for $10 \%-20 \%$ of the phospholipid fatty acid content on average [3]. Metabolites produced by the oxygenation of the arachidonic acid play a key role in the modulation of inflammation [4]. Cyclooxygenase (COX) and lipoxygenase (LOX) enzyme families degrade arachidonic acid to various proinflammatory metabolites. Thromboxane A2, which propagates strong vasoconstriction and platelet aggregation, is synthesized by COX-1 [5]. COX-2 enzyme catalyzes prostacyclin, one of the strongest vasodilator metabo-

Received: September 09, 2017 Accepted: September 21, 2017 Online: August 08, 2018

Correspondence: Dr. Serkan KAHRAMAN. Silivri Devlet Hastanesi, Kardiyoloji Klinigi, Istanbul, Turkey.

Tel: +90 5053825921 e-mail: serkankahraman_86@outlook.com

(c) Copyright 2018 by Istanbul Provincial Directorate of Health - Available online at www.northclinist.com 
lites, synthesis reaction [6]. The second important pathway producing eicosanoids is catalyzed by the LOX enzyme family. Leukotriene B4 produced by this pathway is a known mediator of programmed apoptosis and atherosclerosis [7]. Owing to this close relationship between arachidonic acid metabolites and endothelial homeostasis, these enzymatic pathways deserve great attention.

Aspirin is an important anti-inflammatory drug that directly inhibits the COX enzyme family. It is an effective antithrombotic drug that is used in the primary and the secondary protection in atherothrombotic diseases and complications $[8,9]$. Aspirin resistance plays an important role on atherosclerosis. Two types of aspirin resistance are defined. The occurrence of new cardiovascular events in patients who are using aspirin is defined as "clinical resistance," and the incomplete blockage of platelet activity in vitro is defined as "laboratory resistance" [10]. Since there is a well-known close relationship between atherosclerosis and inflammation, anti-inflammatory drugs inhibiting certain steps of the arachidonic acid pathway and resistance to them become trend topics of cardiology. The main purpose of the present study was to analyze the association between aspirin resistance and extent and severity of coronary atherosclerosis.

\section{MATERIALS AND METHODS}

\section{Patient population}

One hundred patients with suspected coronary artery disease (CAD) due to typical chest pain or positive non-invasive cardiovascular stress testing who underwent cardiac catheterization between April 1, 2013 and November 30, 2013 were included in the study. Exclusion criteria were defined as follows: thrombocytopenia $\left(<100,000 / \mathrm{mm}^{3}\right)$, thrombocytosis $\left(>400,000 / \mathrm{mm}^{3}\right)$, end-stage renal disease, acute or chronic liver failure, hematologic diseases, history of malignant disease, active infection, intolerance or contraindication to aspirin, under treatment of glycoprotein IIb/IIIa inhibitors in the last 3 days, usage of antithrombotic or anticoagulant treatment other than aspirin in the last 30 days, regular use of nonsteroidal anti-inflammatory treatment in the last 3 months, and subjects $<30$ and $>75$ years old. One hundred patients with known or newly diagnosed CAD already using therapeutic doses of aspirin were included.

\section{Study protocol}

This was a prospective observational study. Basic demographic data of patients included age, gender, body mass index (BMI), glomerular filtration rate (GFR), presence of traditional major cardiovascular risk factors (age, sex, hypertension (HT), diabetes, dyslipidemia, family history of premature cardiovascular disease (CVD), and current smoking). The extent and severity of atherosclerosis was analyzed using the Gensini scoring system (GSS) [11]. Thereafter, venous blood samples were collected for biochemical analysis. The study was approved by the local ethics committee of Istanbul Bilim University (no. 44140529/2013-028). All patients were informed about the study. Written informed consent was obtained from the patients.

\section{Angiographic evaluation}

Angiographic evaluations were done by two different experienced cardiologists. The extent and severity of CAD was assessed by the GSS. The Gensini score was calculated by multiplying the severity coefficient assigned to each coronary stenosis according to the degree of luminal narrowing (reductions of $25 \%, 50 \%, 75 \%, 90 \%$, and $99 \%$, and complete occlusion was given Gensini scores of 1, 2, $4,8,16$, and 32 , respectively) by the coefficient identified based on the functional importance of the myocardial area supplied by that segment as follows: left main coronary artery, 5; proximal segment of the left anterior descending coronary artery, 2.5; mid-segment of the left anterior descending coronary artery, 1.5; apical segment of the left anterior descending coronary artery, 1 ; first diagonal branch, 1 ; second diagonal branch, 0.5 ; proximal segment of the circumflex artery, 2.5 (if right coronary artery dominancy existed, 3.5); distal segment of the circumflex artery, 1 (if dominant, 2); obtuse marginal branch, 1; posterolateral branch, 0.5; proximal segment of the right coronary artery, 1 ; mid-segment of the right coronary artery, 1 ; distal segment of the right coronary artery, 1; and posterior descending artery, 1.

\section{Biochemical analysis}

After terminating oral intake for 8-12 h, blood samples that are drawn from the brachial veins of all patients are injected into dry tubes, and samples are centrifuged before biochemical evaluation. Total cholesterol, low-density lipoprotein (LDL), very-low-density lipoprotein, high-density lipoprotein, triglyceride, fasting blood glucose, blood urea nitrogen, creatinine, and complete blood count were measured.

In our study, aspirin resistance is evaluated by the "VerifyNow" system that is an adenosine diphosphate 
(ADP) stimulation method. Aspirin inhibition levels are detected by extracting blood from patients who are taking therapeutic dose aspirin (at least $100 \mathrm{mg} /$ day) after $12-24 \mathrm{~h}$. "VerifyNow" is a system that is based on the stimulation of fibrinogen-coated particles in full blood with citrate by agonists, such as ADP, thrombin receptor-activating peptide, and arachidonic acid, in the mixing compartment. By adding anticoagulated blood into the mixing compartment, platelets are activated, and platelet aggregation occurs after the bonding between GPIIb/IIIa receptors on activated platelets and particles with fibrinogen. After this reaction, the change of light transmissions is defined as aspirin reaction unit (ARU). $\mathrm{ARU}>550$ is considered as aspirin resistance $[12,13]$.

\section{Statistical analysis}

All statistical analyses were performed using the SPSS 16.0 software (SPSS Inc., Chicago, IL, USA). Fitness to normal distribution was analyzed by the KolmogorovSmirnov test. Homogeneity of variances was calculated by the Levene test and the Lilliefors significance correction. Interobserver agreement between two cardiologists was calculated using the Bland-Altman analysis. Differences among two groups were analyzed by the Student's $t$ test or its non-parametric counterpart, Mann-Whitney $\mathrm{U}$ test. Categorical variables were analyzed by either chi-square test or Fisher's exact test where appropriate. Multivariate logistic regression analysis was performed to explore the factors affecting aspirin resistance. Data were expressed as mean \pm standard deviation or median (minimum-maximum) where appropriate. A p value $<0.05$ was considered as statistically significant.

\section{RESULTS}

One hundred patients who underwent coronary angiography with suspected or known CAD and were using aspirin were enrolled in the study. Clinical and demographic characteristics of all subjects are given in Table 1.

Of these 100 patients, 30 (8 female and 22 male) formed the aspirin-resistant group (ARG), and 70 (22 female and 48 male) formed the control group. There were no statistically significant differences in age, smoking, diabetes mellitus, HT, BMI, GFR levels, beta blocker, angiotensin-converting enzyme inhibitor, angiotensin receptor blocker, and statin usage between the ARG and the control group. Only LDL level was significantly higher in the ARG as shown in Table 2.
TABLE 1. Clinical and demographic characteristics of all subjects

$\mathrm{n}=100$ $\%$

Age (years) $62.72 \pm 7.93$

Gender (female) 30

Diabetes mellitus

Hypertension

45

Hyperlipidemia

Smoking

75

95

32

Stent history

There was no statistically significant difference in the GSS between two cardiologists ( $\mathrm{p}=0.76$ ). GSS was significantly higher in the ARG than in the control group $(80.5(36-166)$ vs. $45(2-209) ; \mathrm{p}<0.001)$ as shown in Figure 1.

In addition to this, 26 of a total of 100 patients had first or repetitious percutaneous coronary intervention (PCI) history. There were no acute stent thrombosis patients, 10 (38.5\%) patients had first PCI, and 16 (61.5\%) patients had reintervention due to stent restenosis. The number of PCI patients was significantly higher in the ARG (13 of 30 (43.3\%) ARG vs. 13 of $70(18.6 \%)$ control group; $\mathrm{p}=0.01)$. Furthermore, when we evaluate the 16 reintervention patients, stent restenosis was significantly higher in the ARG (11 of 16 (68.75\%) ARG vs. 5 of $16(31.25 \%)$ control group; $\mathrm{p}=0.016)$ as shown in Table 3 . There were statistically significant differences in LDL levels, GSS, and stent thrombosis between the ARG and the control group. However, multivariate logistic regression analysis revealed that only GSS ( $\mathrm{p}=0.038$; 95\% CI: $1.001-1.026)$ and PCI history $(\mathrm{p}=0.017 ; 95 \%$ CI: 1.182-89.804) were independent risk factors for aspirin resistance as shown in Table 4.

\section{DISCUSSION}

In our recent study, we aimed to investigate the association between aspirin resistance and extent and severity of coronary atherosclerosis. Antiplatelet therapy remains the most important and effective management in the prevention of important clinical complications of atherothrombosis, namely acute coronary events, cerebral vascular accidents, and all other thrombotic events [7]. Aspirin is an important antiplatelet and anti-inflammatory drug that is fairly well analyzed ever. In the meta- 
TABLE 2. Comparison of baseline characteristics of the ARG and control group

\begin{tabular}{|c|c|c|c|c|c|}
\hline \multirow[t]{2}{*}{ Parameter } & \multicolumn{2}{|c|}{$\begin{array}{c}\text { ARG }(n=30) \\
\text { Mean } \pm \text { SD }\end{array}$} & \multicolumn{2}{|c|}{$\begin{array}{c}\text { Control }(n=70) \\
\text { Mean } \pm \text { SD }\end{array}$} & \multirow[t]{2}{*}{$\mathrm{p}$} \\
\hline & $\mathrm{n}$ & $\%$ & $\mathrm{n}$ & $\%$ & \\
\hline Age (years)* & \multicolumn{2}{|c|}{$63.3 \pm 6.70$} & \multicolumn{2}{|c|}{$62.47 \pm 8.43$} & 0.635 \\
\hline Female (gender) $^{\dagger}$ & 8 & 26.7 & 22 & 31.4 & 0.634 \\
\hline Smoking $^{\dagger}$ & 10 & 33.3 & 22 & 31.4 & 0.852 \\
\hline Diabetes mellitus ${ }^{\dagger}$ & 15 & 50 & 30 & 42.9 & 0.511 \\
\hline Hypertension $^{\dagger}$ & 22 & 73.3 & 53 & 75.7 & 0.601 \\
\hline Beta blocker ${ }^{\dagger}$ & 13 & 43.3 & 43 & 61.4 & 0.095 \\
\hline ACE inhibitor ${ }^{+}$ & 7 & 23.3 & 22 & 31.4 & 0.414 \\
\hline Statin $^{+}$ & 10 & 33.3 & 25 & 35.7 & 0.819 \\
\hline $\mathrm{PPI}^{\dagger}$ & 5 & 16.7 & 6 & 8.6 & 0.236 \\
\hline BMI $\left(\mathrm{kg} / \mathrm{m}^{2}\right)^{*}$ & \multicolumn{2}{|c|}{$27.12 \pm 1.29$} & \multicolumn{2}{|c|}{$26.85 \pm 1.51$} & 0.402 \\
\hline GFR $(\mathrm{ml} / \mathrm{min})^{*}$ & \multicolumn{2}{|c|}{$87.08 \pm 27.92$} & \multicolumn{2}{|c|}{$79.92 \pm 18.04$} & 0.129 \\
\hline $\mathrm{LDL}(\mathrm{mg} / \mathrm{dl})^{*}$ & \multicolumn{2}{|c|}{$128.33 \pm 43.40$} & \multicolumn{2}{|c|}{$108.43 \pm 40.02$} & 0.029 \\
\hline
\end{tabular}

ARG: Aspirin resistant group; ACE: Angiotensin converting enzyme; PPI: Proton pump inhibitor; BMI: Body mass index; GFR: Glomerular filtration rate; LDL: Low density lipoprotein; ${ }^{*}$ Student's t test; ${ }^{+}$Chi-square test.

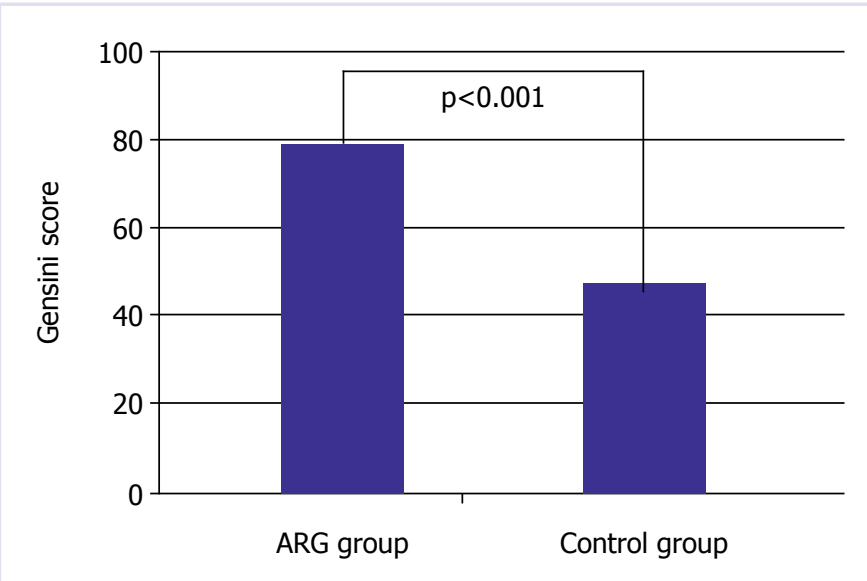

FIGURE 1. Comparison of the Gensini score between the aspirin resistance and non-resistance groups. ARG: aspirinresistant group.

analysis of five randomized studies that included 9853 patients who were followed up with stable CVD, a $21 \%$ decrease in cardiovascular event risk (non-fatal myocardial infarction (MI), non-fatal stroke, and cardiovascular death) and a $13 \%$ decrease in all-cause mortality were found in patients who were taking low dose aspirin (75$325 \mathrm{mg} /$ day) [14]. In a review that included $287 \mathrm{ran}$ domized controlled studies with $>200,000$ patients (An-
TABLE 3. Comparison of angiographic data and aspirin doses of the ARG and control group

\begin{tabular}{|c|c|c|c|}
\hline Parameter & $\begin{array}{c}\text { ARG } \\
(n=30)\end{array}$ & $\begin{array}{l}\text { Control } \\
(n=70)\end{array}$ & $\mathrm{p}$ \\
\hline Aspirin dose (mg/day) & $100(100-300)$ & $100(80-300)$ & 0.018 \\
\hline Gensini score* & $80.5(36-166)$ & 45 (2-209) & $<0.001$ \\
\hline No. of total $\mathrm{PCI}^{\dagger}$ & $13(\% 43.3)$ & $13(\% 18.6)$ & 0.01 \\
\hline No. of reintervention ${ }^{+}$ & $11(68.75 \%)$ & $5(31.25 \%)$ & 0.016 \\
\hline
\end{tabular}

ARG: Aspirin resistant group; PCI: Percutaneous coronary intervention; ${ }^{*}$ Mann Whitney- $U$ test; 'Student's t test.

ti-thrombotic Trialists' Collaboration), a 22\% decrease in the risk of cardiovascular event mortality was detected [9]. The effectiveness of regular aspirin usage in reducing the risk for MI, ischemic stroke, and fatal coronary events among patients with pre-existing atherosclerotic CVDs is well established [15]. Although cheap, effective, and easily accessible, aspirin resistance restricts the usage of this antiplatelet and anti-inflammatory drug. Aspirin resistance is defined as the incapacity of aspirin to decrease platelet production of thromboxane A2, and so platelets activate and aggregate [16]. The prevalence of aspirin resistance has been estimated to be between $5 \%$ 
TABLE 4. Logistic regression analysis giving information about the independent risk factors for aspirin resistance

\begin{tabular}{lccc} 
Parameter & Beta & $\mathrm{p}$ & $\mathrm{CI}(95 \%)$ \\
\hline Gensini score & 0.013 & $\mathbf{0 . 0 4 1}$ & $\mathbf{1 . 0 0 1 - 1 . 0 2 6}$ \\
LDL $(\mathrm{mg} / \mathrm{dl})$ & 0.012 & 0.073 & $0.999-1.025$ \\
PCI history & 2.206 & $\mathbf{0 . 0 3 4}$ & $\mathbf{1 . 1 8 2 - 8 9 . 8 0 4}$ \\
Aspirin dose (mg/day) & -0.009 & 0.062 & $0.991-0.997$ \\
\hline
\end{tabular}

CI: Confidence interval; LDL: Low density lipoprotein; PCI: percutaneous coronary intervention.

and $60 \%$ of aspirin-treated patients for secondary prevention [17]. That is why patients treated with aspirin still retain at substantial risk of clinically important CVDs due to insufficient inhibition of platelet aggregation via the thromboxane A2 pathway. The incidence of aspirin resistance was found to be $30 \%$ in our study, which is compatible with previous ones. It is obvious that patients with aspirin resistance are prone to atherothrombotic and atherosclerotic events. Krasopoulus et al. reported that long-term aspirin-treated patients who are resistant to aspirin are at a greater risk of important cardiac morbidity than patients who are sensitive to aspirin [18].

As we mentioned previously, atherosclerosis is a chronic low-grade inflammatory state. Aspirin, due to the COX enzyme inhibitor activity, is also a well-known anti-inflammatory drug. The influence of inflammation on the progression of atherosclerosis and rupture of atherosclerotic plaque opens a new therapeutic era for atherosclerosis. Not only aspirin but also some other drugs, such as statins, thiazolidinediones (glitazones), and renin-angiotensin aldosterone system blockers, exert their anti-atherosclerotic effect through the modulation of endothelial inflammation $[19,20]$. In addition to this, in a recent study, it was found that antiplatelet agents, namely aspirin, clopidogrel, or ticagrelor, significantly reduce high sensitive $C$-reactive protein level, which is a key biomarker of inflammation [21]. In our study, there was no significant difference in statin and renin-angiotensin aldosterone system blocker usage between the ARG and the control group that can affect the inflammatory state. Furthermore, in a similar study, Li et al. showed that the anti-inflammatory effect of tanshinone IIA, one of the most abundant constituents of the root of the red sage, improves inflammation and increases atherosclerotic plaque stability [22]. In light of foregoing data, it is known that atherosclerosis is one of the reasons of in- flammatory state, and anti-inflammatory agents, such as aspirin, could exert anti-atherosclerotic effect. We might conclude that patients with aspirin resistance could be more prone to atherosclerosis and atherothrombosis. As far as we see, there is hardly any literature assessment to analyze the relationship between GSS and aspirin resistance. In our study, we revealed that the GSS was significantly higher in aspirin-resistant patients, meaning that atherosclerotic burden is significantly higher in aspirin resistance. Furthermore, we found that coronary reintervention ratio is significantly higher in the ARG. We consider that aspirin has antiplatelet and anti-inflammatory effects, and in aspirin resistance patients, lack of these effects is the possible reasons of high GSS and coronary reintervention ratio.

Although the possible mechanisms of aspirin resistance are beyond the scope of this article, the effect of aspirin dose on aspirin resistance could be discussible. Actually, Gengo et al. demonstrated that patients who are non-responsive to $81 \mathrm{mg} /$ day dose of aspirin become responsive at $162 \mathrm{mg} /$ day or at a greater dose [23]. In a similar study, Duzenli et al. revealed that increasing the aspirin dose to $300 \mathrm{mg} /$ day or adding clopidogrel to aspirin can provide adequate platelet inhibition in a significant number of patients with impaired responses to low dose aspirin [24]. Interestingly, in our study, the mean aspirin dose in the control group was significantly higher than that in the ARG. In previous studies, non-responsive patients became aspirin responsive at doses $>150$ $\mathrm{mg} /$ day. In our study, although both groups' aspirin doses were in therapeutic ranges, they were not in maximal doses. This could be the possible reason of this result.

The prothrombotic and inflammatory state is related with aspirin resistance in patients with hyperlipidemia, and it is known that this relationship does not depend on LDL cholesterol levels. In our study, LDL level was significantly higher in the ARG. However, there were some other factors, such as statin usage, and other causes of inflammatory state could affect aspirin resistance [25].

\section{Conclusion}

Our study concluded that atherosclerotic burden as calculated by the GSS is significantly higher in aspirin-resistant patients. According to this result, we suggest that aspirin treatment can be prescribed in higher doses in aspirin resistance patients with coronary events. Furthermore, Gensini score and PCI history could be independent predictors of aspirin resistance. Absolutely higher 
scaled studies are needed to further elucidate the clinical implications of these findings.

\section{Limitations of the study}

One of the limitations was the lack of basal ARU before aspirin treatment. However, all the patients were evaluated by VerifyNow, and ARU levels were obtained under therapeutic dose aspirin treatment, with a cut-off level of 550, which is considered as a critical level in most studies. This provides us to get over this limitation in a way. Another limitation was the small number of subjects in the ARG. In addition, low dose aspirin was used in the ARG than the control group, but both of them were in therapeutic ranges.

Conflict of Interest: The authors declare no conflict of interest.

Financial Disclosure: The authors declared that this study has received no financial support.

Authorship Contributions: Concept - S.K. A.D. C.C.; Design S.K., A.D., C.C.; Supervision - S.K., A.D., C.C.; Materials - S.K., M.Z., E.U., O.D., C.C.; Data collection \&/or processing - S.K., M.Z., E.U., O.D., C.C.; Analysis and/or interpretation - S.K., A.D., C.C.; Writing - S.K., A.D., E.U., O.D., C.C.; Critical review - S.K., A.D., M.Z., C.C.

\section{REFERENCES}

1. Mattmiller SA, Carlson BA, Sordillo LM. Regulation of inflammation by selenium and selenoproteins: impact on eicosanoid biosynthesis. J Nutr Sci 2013;2:e28.

2. Mathers CD, Loncar D. Projections of global mortality and burden of disease from 2002 to 2030. PLoS Med 2006;3:e442. [CrossRef]

3. Smith GI, Atherton P, Reeds DN, Mohammed BS, Rankin D, Rennie $\mathrm{MJ}$, et al. Omega-3 polyunsaturated fatty acids augment the muscle protein anabolic response to hyperinsulinaemia-hyperaminoacidaemia in healthy young and middle-aged men and women. Clin Sci (Lond) 2011;121:267-78. [CrossRef]

4. Samuelsson B. Arachidonic acid metabolism: role in inflammation. Z Rheumatol 1991;50:3-6.

5. Dogné JM, de Leval X, Hanson J, Frederich M, Lambermont B, Ghuysen A, et al. New developments on thromboxane and prostacyclin modulators part I: thromboxane modulators. Curr Med Chem 2004;11:1223-41. [CrossRef]

6. Catella-Lawson F, McAdam B, Morrison BW, Kapoor S, Kujubu D, Antes L, et al. Effects of specific inhibition of cyclooxygenase-2 on sodium balance, hemodynamics, and vasoactive eicosanoids. J Pharmacol Exp Ther 1999;289:735-41.

7. Moroney MA, Alcaraz MJ, Forder RA, Carey F, Hoult JR. Selectivity of neutrophil 5-lipoxygenase and cyclo-oxygenase inhibition by an anti-inflammatory flavonoid glycoside and related aglycone flavonoids. J Pharm Pharmacol 1988;40:787-92. [CrossRef]

8. Collaborative overview of randomised trials of antiplatelet therapy. I: Prevention of death, myocardial infarction, and stroke by prolonged antiplatelet therapy in various categories of patients. Antiplatelet Trialists' Collaboration. BMJ 1994;308:81-106. [CrossRef]
9. Antithrombotic Trialists' Collaboration. Collaborative meta-analysis of randomised trials of antiplatelet therapy for prevention of death, myocardial infarction, and stroke in high risk patients. BMJ 2002;324:71-86.

10. Kuliczkowski W, Witkowski A, Polonski L, Watala C, Filipiak K, Budaj $\mathrm{A}$, et al. Interindividual variability in the response to oral antiplatelet drugs: a position paper of the WorkingGroup on antiplatelet drugs resistance appointed by the Section of Cardiovascular Interventionsof the Polish Cardiac Society, endorsed by the Working Group on Thrombosis of the EuropeanSociety of Cardiology. Eur Heart J 2009;30:426-35.

11. Gensini GG. A more meaningful scoring system for determining the severity of coronary heart disease. Am J Cardiol 1983;51:606. [CrossRef]

12. Gachet C, Aleil B. Testing antiplatelet therapy. Eur Heart J Suppl 2008;10: A28-A34. [CrossRef]

13. Michelson AD. Methods for the measurement of platelet function. Am J Cardiol 2009;103:20A-26A. [CrossRef]

14. Berger JS, Brown DL, Becker RC. Low-dose aspirin in patients with stable cardiovascular disease: a meta-analysis. Am J Med 2008;121:43-9.

15. Antithrombotic Trialists' (ATT) Collaboration, Baigent C, Blackwell L, Collins R, Emberson J, Godwin J, et al. Aspirin in the primary and secondary prevention of vascular disease: collaborative meta-analysis of individual participant data from randomised trials. Lancet 2009;373:1849-60. [CrossRef]

16. Hankey GJ, Eikelboom JW. Aspirin resistance. Lancet 2006;367:60617. [CrossRef]

17. Heistein LC, Scott WA, Zellers TM, Fixler DE, Ramaciotti C, Journeycake JM, et al. Aspirin resistance in children with heart disease at risk for thromboembolism: prevalence and possible mechanisms. Pediatr Cardiol 2008;29:285-91. [CrossRef]

18. Krasopoulos G, Brister SJ, Beattie WS, Buchanan MR. Aspirin "resistance" and risk of cardiovascular morbidity: systematic review and meta-analysis. BMJ 2008;336:195-8. [CrossRef]

19. Sukhova GK, Williams JK, Libby P. Statins reduce inflammation in atheroma of nonhuman primates independent of effects on serum cholesterol. Arterioscler Thromb Vasc Biol 2002;22:1452-8. [CrossRef]

20. Husain K, Hernandez W, Ansari RA, Ferder L. Inflammation, oxidative stress and renin angiotensin system in atherosclerosis. World J Biol Chem 2015;6:209-17. [CrossRef]

21. Layne K, Di Giosa P, Ferro A, Passacquale G. LB03.03: Ant1-1nflammatory effects of ant1-platelet drugs: implication for atherosclerosis. J Hypertens 2015;33:e126. [CrossRef]

22. Li Y, Guo Y, Chen Y, Wang Y, You Y, Yang Q, et al. Establishment of an interleukin-1 $\beta$-induced inflammation-activated endothelial cell-smooth muscle cell-mononuclear cell co-culture model and evaluation of the anti-inflammatory effects of tanshinone IIA on atherosclerosis. Mol Med Rep 2015;12:1665-76. [CrossRef]

23. Gengo F, Westphal ES, Rainka MM, Janda M, Robson MJ, Hourihane JM, et al. Platelet response to increased aspirin dose in patients with persistent platelet aggregation while treated with aspirin $81 \mathrm{mg}$. J Clin Pharmacol 2016;56:414-21. [CrossRef]

24. Duzenli MA, Ozdemir K, Aygul N, Soylu A, Tokac M. Comparison of increased aspirin dose versus combined aspirin plus clopidogrel therapy in patients with diabetes mellitus and coronary heart disease and impaired antiplatelet response to low-dose aspirin. Am J Cardiol 2008;102:396-400. [CrossRef]

25. Cipollone F, Mezzetti A, Porreca E, Di Febbo C, Nutini M, Fazia M, et al. Association between enhanced soluble CD40L and prothrombotic state in hypercholesterolemia: effects of statin therapy. Circulation 2002;106:399-402. [CrossRef] 